\title{
Preparation of Autofluorescent Polyacrylamide Nanoparticles
}

\author{
Qiao Wang ${ }^{1, a}$, Jinke Wang1, ${ }^{\text {b, }}$ \\ 1 State Key Laboratory of Bioelectronics, Southeast University, Nanjing 210096, China \\ aE-mail address: 220151647@seu.edu.cn \\ bE-mail address: wangjinke@seu.edu.cn \\ ${ }^{*}$ Correspondence should be addressed to Jinke Wang
}

Keywords: Polyacrylamide Nanoparticles, Glutaraldehyde, Near infrared, Fluorescence.

\begin{abstract}
Nowadays, tumor imaging is widely used in biomedical field. However, the materials of imaging is rarely and strict. This study developed a method for preparing autofluorescent polyacrylamide nanoparticles (NPs). The polyacrylamide NPs were treated with glutaraldehyde, and then removed excess glutaraldehyde through ultrafiltration, glycine neutralization and ethanol precipitation, respectively. Based on this method, the polyacrylamide NPs showed strong nearinfrared fluorescence (NIRF) at the emission wavelength of both 720 and $820 \mathrm{~nm}$. Additionally, the nanoparticles were efficiently internalized into RAW264.7 cells and displayed strong green and red fluorescence observed with fluorescence microscope. Therefore, the NPs are expected to be applied to tumor imaging in future.
\end{abstract}

\section{Introduction}

In recent years, optical imaging is widely used in medical diagnosis and molecular biology, which will have an important impact on the prevention and treatment of cancer and other deadly diseases [1, 2]. However, it is necessary to find a fluorescein carrier with good biocompatibility [3]. The polyacrylamide NPs are an important water-soluble polymeric material, which can form a stable colloidal dispersion system in water, showing excellent biocompatibility [4]. Furthermore, in the cross-link process of using the carrier to construct the fluorescence probe, which usually affects the intrinsic properties of fluorescent dyes and carriers. Therefore, it is necessary to explore a material with spontaneous fluorescence properties. Many researchers have used glutaraldehyde to construct organic nanomaterials with spontaneous fluorescence properties [5-8], but their emission fluorescence can not meet the imaging needs for only visible range. The paper constructed a spontaneous fluorescence polyacrylamide NPs with NIFR properties, indicating potential wide application in the optical imaging.

\section{Methods}

\subsection{Preparation of polyacrylamide NPs}

Polyacrylamide NPs were prepared by reversed-phase microemulsion system. $90 \mathrm{~mL}$-hexane was firstly add to a dry $250 \mathrm{~mL}$ round bottom flask, and stirred in argon for $10 \mathrm{~min}$. Then add $3.2 \mathrm{~g}$ surfactant AOT and $6.3 \mathrm{~mL}$ Brij30, stir mixture for 40 minutes under argon protection. Adding $80 \mu \mathrm{L}$ new prepared ammonium persulfate (APS) aqueous solution $(10 \% \mathrm{w} / \mathrm{v})$ and $80 \mu \mathrm{L}$ TEMED initiation polymerization. After that add the monomer solution, which includes $530 \mathrm{mg}$ acrylamide, $30 \mathrm{mg}$ APMA, $160 \mathrm{mg}$ MBA and $2 \mathrm{ml}$ ultra-pure water, to the flask and the reaction was $3 \mathrm{~h}$ under the protection of argon. After the polymerization reaction, transfer to the rotary evaporator to remove nhexane. The final nanoparticle suspension was obtained through ethanol precipitation and filtration. The hydrodynamic size and zeta potential of polyacrylamide NPs suspension were analyzed using the Zetasizer Nano particle analyzer. 


\subsection{Glutaraldehyde deals with polyacrylamide NPs}

The addition of $400 \mu \mathrm{L}$ glutaraldehyde (25\%) was added to the $1600 \mu \mathrm{L}$ nanoparticles, ultrasonic reaction under $37{ }^{\circ} \mathrm{C}$ for $1 \mathrm{~h}$, After ultrasound, pour into the dialysis bag, dialysis $12 \mathrm{~h}$. The above treated samples were concentrated in the ultrafiltration tube, and then the $5500 \mathrm{rpm}$ centrifuge was removed for $30 \mathrm{~min}$ (the supernatant is named S1). In addition, add $2000 \mu \mathrm{L}$ glycine solution (1 M), $1500 \mu \mathrm{L} \mathrm{H} \mathrm{H}_{2} \mathrm{O}$. After $37^{\circ} \mathrm{C}$ water bath overnight, with ethanol precipitation except the supernatant (S2), with $4000 \mu \mathrm{L}$ water hung heavy precipitation final suspension (NPs-f).

\subsection{Transfection cells}

First suck out cell culture from the orifice plate, and then clean with PBS 2-3 times, add $500 \mu \mathrm{L} /$ hole cell cultures, and add $100 \mu \mathrm{L}$ nanoparticles to the corresponding hole, incubate at $37{ }^{\circ} \mathrm{C}$ for 4 hours. After the cell was fixed at room temperature for 20 min with $4 \%$ paraformaldehyde, DAPI staining was used to dye the nucleus, and then the cells were imaged with fluorescence microscopy.

\section{Results}

\subsection{Characterization of polyacrylamide NPs}

The polyacrylamide NPs possessed a great dispersibility through the transmission electron microscopy (TEM) observation (Figure 1A). The average particle size in aqueous solution was about $79 \mathrm{~nm}$ with dynamic light scattering (DLS) in a particle size analyzer (Figure 1B). This nanoparticle can be used for subsequent experiments for their great dispersibility and size uniformity in water.

\subsection{Glutaraldehyde deals with polyacrylamide NPs}

The polyacrylamide NPs treated by glutaraldehyde presented deeper yellow under the white light (Figure 2A). The NPs showed slight yellow with the ultra-filtered and glycine added (Figure 2B). Novelly, the NPs displayed strong NIFR at the emission wavelength of both 720 and $820 \mathrm{~nm}$ (Figure 2C). Additionally, it was found that the NIRF at the emission wavelength of $720 \mathrm{~nm}$ was much stronger than that at $820 \mathrm{~nm}$ (Figure 2C).

\subsection{Cellular internalization}

The cellular internalization of the nanoparticle was detect by the common fluorescence microscopy, It was found that the nanoparticles could be efficiently internalized into RAW264.7 cells and displayed strong green and red fluorescence with fluorescence microscope, respectively (Figure 3). The results proved that the cells labeled by polyacrylamide NPs showed good imaging effect with both visible and infrared fluorescence.

A

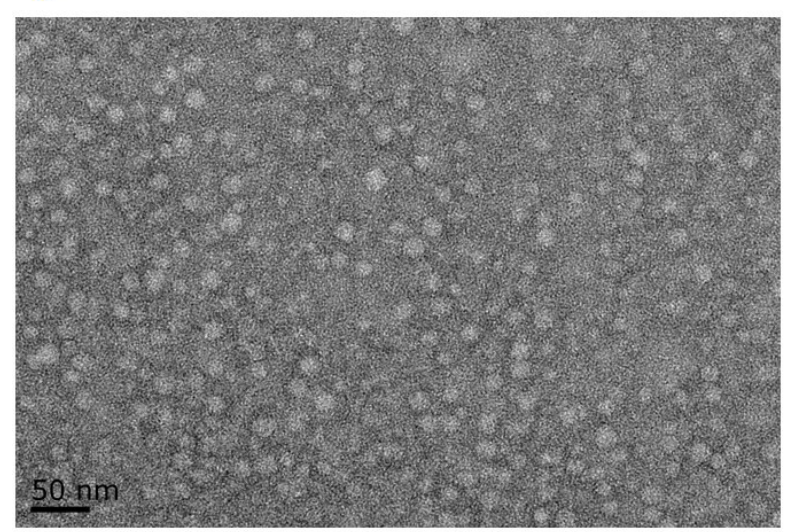

B

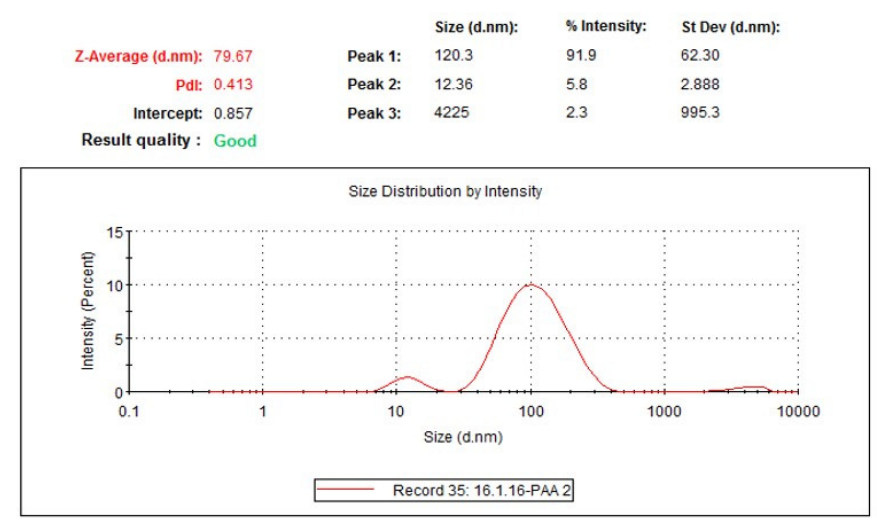

Figure 1

Fig.1 Characterization of polyacrylamide NPs with SEM and DLS 

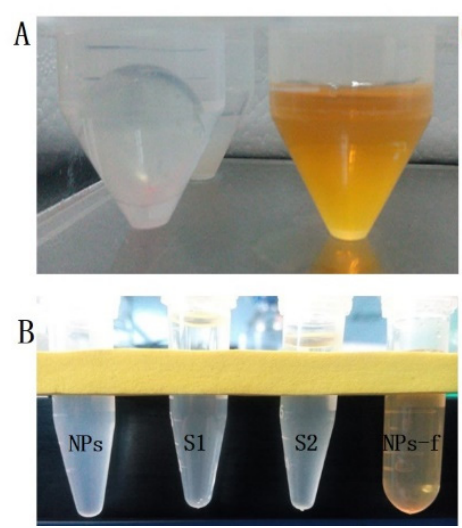

Figure 2
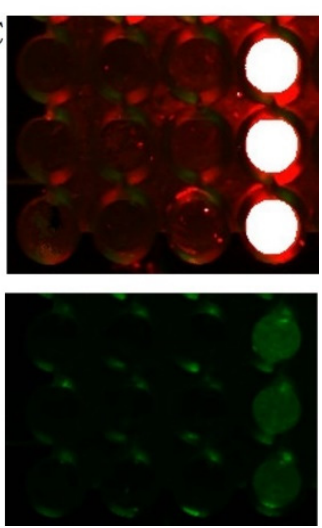
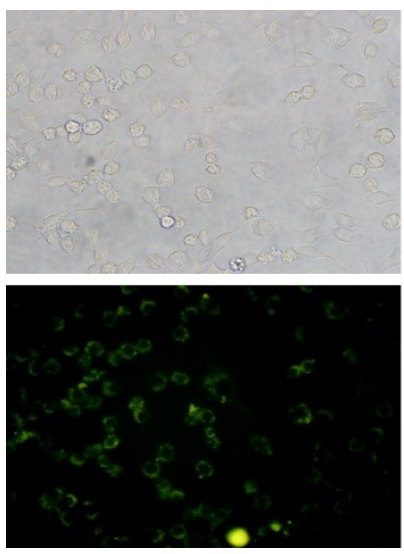

Figure 3
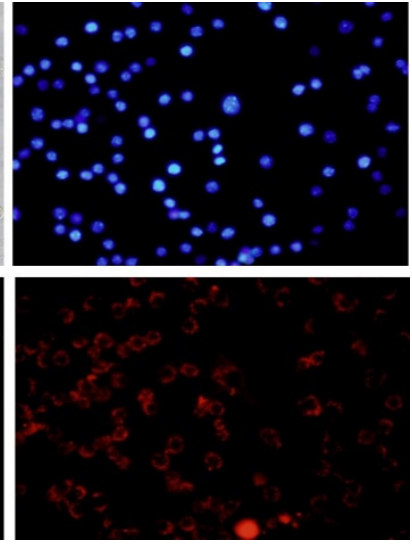

Fig.2 (A) The NPs and the NPs were treated with glutaraldehyde and glycine. (B) NPs: Untreated nanoparticles; S1: Ultra-filtered supernatant; S2: The supernatant of ethanol precipitation treatment; NPs-f: The final treated nanoparticles.

(C) Appearance of NPs, S1, S2 and NPs-f solution, and NIRF imaging at 720 and $820 \mathrm{~nm}$ emission wavelength.

Fig.3 Treatment of RAW264.7 cells with polyacrylamide NPs for $4 \mathrm{~h}$. The cell nuclei were then stained by DAPI and the cells were imaged at the bright field. Microscope images of DAPI, green fluorescence, red fluorescence were obtained, respectively.

\section{Conclusion}

This study developed a method for preparing autofluorescent polyacrylamide NPs, which successfully prepared polyacrylamide NPs. The nanoparticles were efficiently internalized into RAW264.7 cells and displayed strong green and red fluorescence with fluorescence microscope, respectively. Moreover, It was found that polyacrylamide NPs possessed NIR autofluorescence (NIRF) in addition to their visible fluorescence, which is critical for in vivo application of the nanomaterial, indicating a wide potential biomedical applications in future.

\section{Acknowledgments}

This research was financially supported by the National Natural Science Foundation of China (Grant NO. 61571119).

\section{References}

[1] Ntziachristos V. Fluorescence molecular imaging [J]. Annual Review of Biomedical Engineering, 2006, 8(8):1-33.

[2] Luker G D, Luker K E. Optical imaging: current applications and future directions [J]. Journal of Nuclear Medicine Official Publication Society of Nuclear Medicine, 2008, 49(1):1-4.

[3] Jr O K. Clinical importance of biocompatibility and its effect on haemodialysis treatment [J]. Nephrol Dial Transplant, 2003, 18 Suppl 5(4):v41.

[4] Gupta A, Wang S, Marko A, et al. Polyacrylamide-Based Biocompatible Nanoplatform Enhances the Tumor Uptake, PET/fluorescence Imaging and Anticancer Activity of a Chlorophyll Analog [J]. Theranostics, 2014, 4(6):614-28.

[5] Allouche J. Synthesis of Organic and Bioorganic Nanoparticles: An Overview of the Preparation Methods [M]// Nanomaterials: A Danger or a Promise Springer London, 2013:27-74.

[6] Sailaja A K, Amareshwar P, Chakravarty P. Different technique used for the preparation of nanoparticles using natural polymers and their application [J]. International Journal of Pharmacy \& Pharmaceutical Sciences, 2011, 3:45-50. 
[7] Sultana F, Manirujjaman, Imran-Ul-Haque, et al. An overview of nanogel drug delivery system [J]. Journal of Applied Pharmaceutical Science, 2013, 3(8):S95-S105.

[8] Shin d H, Heo M B, Lim Y T. Self-assembled polyelectrolyte nanoparticles as fluorophore-free contrast agents for multicolor optical imaging [J]. Molecules, 2015, 20(3):4369-82. 